\title{
Turbulence-Flame Interactions - the Mechanisms of Flame Strain and Wrinkling
}

\author{
Adam M. Steinberg* James F. Driscoll ${ }^{\dagger}$ \\ Steven L. Ceccio $\ddagger$ \\ University of Michigan, Ann Arbor, MI, 48109, USA
}

\begin{abstract}
Experimental measurements taken using Cinema-Stereoscopic Particle Image Velocimetry (CS-PIV) are used to investigate the phenomena responsible for the creation of flame surface area and flame wrinkles in turbulent premixed combustion. This diagnostic provides three component velocity fields and the flame front location at rates of over $1 \mathrm{kHz}$ with a spatial resolution of $140 \mu \mathrm{m}$. Previous results (Steinberg et al., Proc. Combust. Inst., 32, 2008) have shown that characterizing turbulence-flame interactions using vorticity does not accurately describe the strain rate exerted on a flame surface. This observation is in conflict with the typical idea that strain on a flame is exerted by vortices. Hence, an alternative description of the turbulence-flame interaction was sought. Decomposition of the velocity gradient field indicates that the turbulent structures responsible for the generation of flame surface area are those of concentrated fluid dynamic strain rate, not vorticity. Hence, a new interpretation of the stretching process is presented that involves the simultaneous straining of the flame by turbulent strain-rate structures and redistribution of the flame surface into wrinkles by vortical structures. This interpretation is confirmed using the CS-PIV measurements. By observing the interaction between various turbulent structures and the flame, it is shown that the generation of flame surface area is caused by strong strain-rate structures interacting with the flame. These structures provide a good characterization of the strength, residence time, and location of the flame surface generation. Furthermore, it is shown that the strain-rate structure interpretation is more versatile in describing the complex geometries of turbulence-flame interactions than the canonical vortex ideas. Hence, models for the generation of flame surface area by turbulence should be based on turbulent strain-rate structures, not vortices.
\end{abstract}

\section{Introduction}

The use of turbulent premixed flames to reduce $\mathrm{NO}_{x}$ from combustion devices such as gas turbine engines has become a popular method of meeting increasingly stringent emission requirements. However, the accurate simulation of such flames is still a challenge and improved models are required to design the efficient, clean, and robust engines of the future. In this mode of combustion, reactions typically occur in thin corrugated sheets that are similar in structure to dynamically stretched laminar flames. The area of these sheets or 'flamelets' largely determines the rate at which fuel is consumed, heat is liberated, and the turbulent flame propagates. Hence, an accurate model for the manner in which turbulence generates flame surface area is required.

One promising approach is to employ the flame surface density transport equation:

$$
\frac{\partial \Sigma}{\partial t}+\nabla \cdot\left[\left(\vec{u}+S_{L} \hat{n}\right) \Sigma\right]=(-\hat{n} \cdot(\hat{n} \cdot \nabla) \vec{u}+\nabla \cdot \vec{u}) \Sigma+\left(S_{L} \nabla \cdot \hat{n}\right) \Sigma+D
$$

Here, $\vec{u}$ is the velocity field, $\hat{n}$ is the flame surface normal vector pointing into the reactants, and $S_{L}$ is the propagation speed of the flame (i.e. the laminar flame speed). The flame surface density $(\Sigma)$ is defined as

\footnotetext{
${ }^{*}$ Research Assistant, Department of Aerospace Engineering, 2041 FXB Building. Member AIAA.

${ }^{\dagger}$ Professor, Department of Aerospace Engineering, 3004 FXB Building. Fellow AIAA.

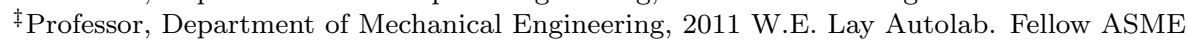


the flame surface area per unit volume. The left side of this equation represents the advection, convection, and normal propagation of the flame surface. The right side represents the mechanisms that generate and destroy flame surface area. The first term describes the straining of the flame due to velocity gradients, while the second accounts for propagation of the curved wave. An additional destruction term, $D$, is often added to account for effects such as quenching and flamelet interaction.

The flame surface density equation is of central importance in many RANS and LES models of turbulent premixed combustion. For example, it is used to close the reaction rate equations in the Coherent Flamelet Model. ${ }^{1,2}$ In LES studies, it is often used to determine the amount of flame surface area at subgrid scales. ${ }^{3-5}$ However, in all cases sub-models for the three unclosed terms on the right side of Eqn. 1 must be invoked. Of these, the strain rate:

$$
a_{t}=-\hat{n} \cdot(\hat{n} \cdot \nabla) \vec{u}+\nabla \cdot \vec{u}
$$

represents the transport of flame surface area due to turbulence. Hence it must describe the interaction of a flow field containing turbulent structures of various 'size', 'strength', 'geometry', and 'nature' with a dynamically stretched flame front. This statement, while quite ambiguous, indicates many of the difficulties in modeling this process. In the past, the wrinkling of a flame due to turbulence has been envisioned to be the result of a set of simple theoretical vortices interacting with the flame front. These deterministic structures had an easily definable scale and strength that could be used to characterize the interaction. However, the use of preconceived vortices as the building block mechanism for turbulence-flame interactions is very restrictive and may not accurately represent the nature of the true interactions responsible for flame strain.

\section{The Straining of Turbulent Premixed Flamelets}

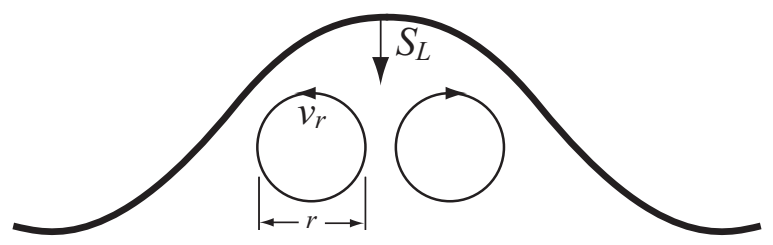

(a) Canonical vortex interpretation

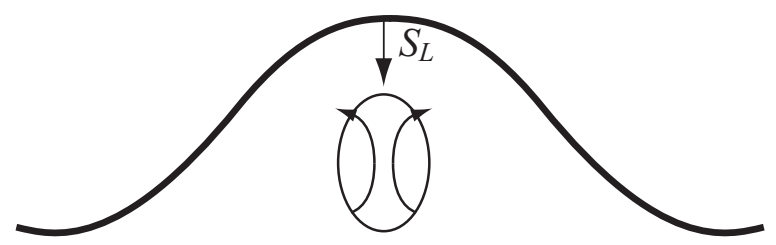

(b) Strain interpretation

Figure 1. Generation of flame surface area.

The straining of a turbulent premixed flame involves the interaction between turbulent structures and the flame front. These structures are defined as a local volume in which a particular turbulence variable is concentrated around a peak. In order to investigate this interaction, Poinsot et al. ${ }^{6}$ considered the configuration shown in Fig. 1(a) in which a pair of two-dimensional vortices (structures of concentrated vorticity) were impinged on a laminar flame. Interactions were carried out over a range of vortex sizes and strengths, which were analyzed by Meneveau and Poinsot ${ }^{7}$ to deduce the effect of an entire turbulent flow.

This configuration has been studied in great detail, both computationally and experimentally. Colin et al. ${ }^{8}$ and Charlette et al. ${ }^{4}$ extended the work of Meneveau and Poinsot ${ }^{7}$ by considering additional parameters and different methods of summing the scales. Furthermore, it is a popular configuration for the study of flame quenching, baroclinic effects on vorticity, and flame stability. ${ }^{9,} 10$ Extensive reviews of the canonical flame-vortex interaction are provided by Renard et al. ${ }^{11}$ and Kadowaki and Hasegawa. ${ }^{12}$ However, this picture of turbulence-flame interactions can be misleading. As shown by Steinberg et al. ${ }^{13}$ vortices in the canonical configuration do not characterize the strain rate exerted on a flame front; vortex pairs of similar strength and size exerted different strain rates and for different lengths of time. Hence, a different mechanism for the straining of a flame front due to turbulence was sought.

Consider the strain rate defined by Eqn. 2. In incompressible turbulence, the strain rate exerted by the turbulence is given by the first term on the right. The second term represents the strain induced by the dilation of the gas through the flame. Hence, the turbulence contribution depends on the flame orientation and the velocity gradient tensor, which can be written as:

$$
\nabla \vec{u}=\frac{\partial u_{i}}{\partial x_{j}}=\frac{1}{2}\left(\frac{\partial u_{i}}{\partial x_{j}}+\frac{\partial u_{j}}{\partial x_{i}}\right)+\frac{1}{2}\left(\frac{\partial u_{i}}{\partial x_{j}}-\frac{\partial u_{j}}{\partial x_{i}}\right)=S_{i j}-\Omega_{i j}
$$


where $\underline{S}$ is the symmetric strain rate tensor and $\underline{\Omega}$ is the antisymmetric rotation rate tensor. The elements of $\underline{\Omega}$ are the various vorticity $(\vec{\omega})$ components. However, the antisymmetry of the rotation rate tensor means that $\hat{n} \cdot(\hat{n} \cdot \underline{\Omega})=0$ and the strain rate induced on the flame can be written as:

$$
a_{t}=-\hat{n} \cdot(\hat{n} \cdot \underline{S})+\nabla \cdot \vec{u}=\left(\delta_{i j}-n_{i} n_{j}\right) S_{i j}
$$

where $\delta_{i j}$ is the Kronecker delta function. Hence, the strain rate exerted on the flame does not involve vorticity, but is directly related to the fluid strain rate tensor. This indicates that velocity gradients appearing as fluid dynamic strain rate generate flame surface area. A flow containing only vorticity and no strain rate should not strain the flame.

Thus is is apparent that the image of rotating 'vortices' straining the flame to generate area is misconceived. These a priori determined structures are not a proper representation of the structures responsible for straining the flame surface. Furthermore, attempting to associate a characteristic strain rate with a radius and rotational velocity of the vortical structures may lead to misinterpreted results. We must then ask: what turbulent structures lead to the generation of flame surface area? The answer is regions of concentrated fluid dynamic strain rate (strain-rate structures). That is, a turbulent flow consists of evolving and interacting vorticity and strain rate fields in which their are coherent volumes both of high vorticity (vortices) and high fluid dynamic strain rate (strain-rate structures). It is these latter regions that are responsible for the generation of flame surface area.

The importance of strain-rate structures in non-reacting turbulence has been studied by many authors. ${ }^{14-17}$ Also, the effect of the strain-rate field on scalar gradients in a turbulent flame has been investigated. ${ }^{18,19}$ However, the role of coherent strain-rate structures in the generation of flame surface area has not yet been studied. Considering such structures, the canonical picture of turbulence-flame interactions in Fig. 1(a) is modified to that of Fig. 1(b). In this view, a single extensionally straining structure is responsible for the generation of flame surface area.

This description avoids many of the issues associated with the traditional counter-rotating vortices. Firstly, the restrictive building block of an a priori constructed geometry is eliminated. Instead, individual strain-rate structures that may be of any orientation, configuration, or geometry affect the flame surface area. This negates the need to consider strain rate patterns associated with different vortex configurations. Secondly, it allows for proper characterization of the size and strength of the important turbulent structures. Instead of a vortex radius and rotational velocity, parameters associated with the strain-rate structure should be used. Finally, important structures in real turbulence can be identified and studied. Studying real turbulence allows the structures to evolve naturally, provides a rich spectrum of interaction configurations, and allows complex phenomena such as turbulence induced flame instabilities to be observed.

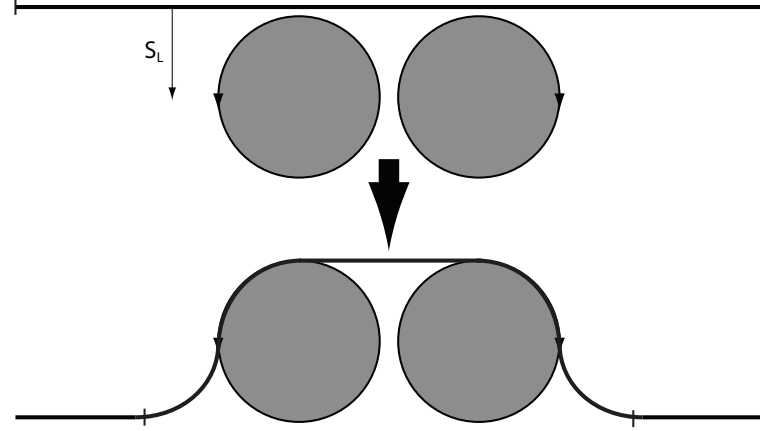

(a) Solid body rotations. A wrinkle forms but no flame surface is generated.

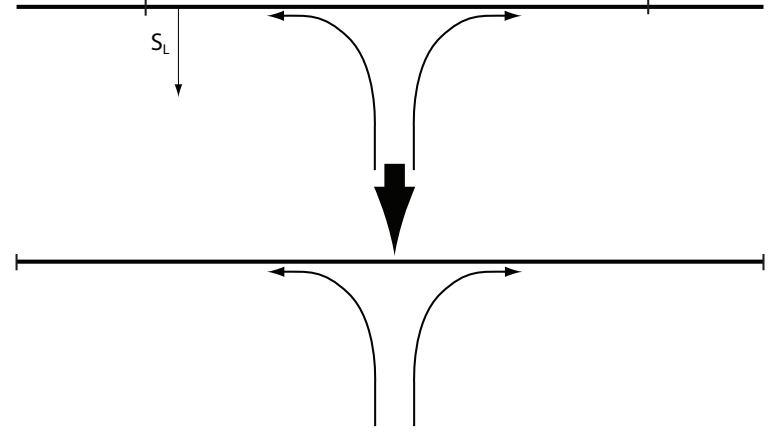

(b) Straining structure (counterflow). No wrinkle forms, but flame surface is generated.

Figure 2. Interactions between different forms of turbulent structure.

At this point it is interesting to consider the effect of a pure vortical or pure strain rate flow field interacting with a flame. The former corresponds to a solid body rotation, while the latter commonly occurs in a counterflow geometry. The flame front is considered to be an infinitely long propagating surface. Hence the interaction is analogous to a local turbulence-flame interaction in a much larger flame. The solid body rotations (Fig. 2(a)) are configured in the canonical (vortex pair) manner. As they pass through the flame, a wrinkle is formed. However, this is a process of simply wrapping up existing flame surface and no additional 
area is created; the length between the hashes does not change. Conversely, the straining flow field does not create a flame wrinkle, but produces a flat flame with greater surface area as shown in Fig. 2(b); the hash marks move apart. Hence, the interaction between a turbulent flow field and a flame front is a combined processes of strain-rate structures generating flame surface area and vortical structures redistributing it into wrinkles. Furthermore, both processes require the flame surface and turbulent structures to be oriented in such a manner that the velocity gradients are effective. This is clearly seen for the strain rate components in Eqn. 4; if $\hat{n}$ is not properly oriented, the $\underline{S}$ components have no effect on $a_{t}$. Similarly, if the solid body rotations in Fig. 2(a) encountered a flame segment already wrinkled in the final configuration, no additional wrinkling would occur.

Recognizing that different turbulent structures have different roles in the interaction, it is important to understand the evolution of both the strain rate and vorticity fields and their relationship. The transport equation for the vorticity in a reacting flow has previously been derived several authors: $:^{9} 20$

$$
\frac{D \omega_{i}}{D t}=S_{i k} \omega_{k}+\nu \frac{\partial^{2} \omega_{i}}{\partial x_{k} \partial x_{k}}+\frac{1}{\rho^{2}}\left(\epsilon_{i j k} \frac{\partial p}{\partial x_{j}} \frac{\partial \rho}{\partial x_{k}}\right)+\xi
$$

where $\epsilon_{i j k}$ is the Levi-Civita tensor. A similar equation has been derived for the strain rate in a non-reacting flow, and can be extended to a reacting flow (see the Appendix):

$$
\frac{D S_{i j}}{D t}=S_{i k} S_{k j}-\frac{1}{4}\left(\omega_{i} \omega_{j}-\delta_{i j} \omega_{k} \omega_{k}\right)+\nu \frac{\partial^{2} S_{i j}}{\partial x_{k} \partial x_{k}}-\frac{1}{\rho} \frac{\partial^{2} p}{\partial x_{i} \partial x_{j}}+\frac{1}{2 \rho^{2}}\left(\frac{\partial p}{\partial x_{i}} \frac{\partial \rho}{\partial x_{j}}+\frac{\partial p}{\partial x_{j}} \frac{\partial \rho}{\partial x_{i}}\right)+\zeta
$$

The terms $\xi$ and $\zeta$ represents the effects of viscosity gradients. As can be seen, the evolution of the vorticity and strain rate fields are mutually interacting. However, the existence of a particular vorticity field at a given time does not imply that a particular strain rate field must exist or vice versa. Hence, while the replacement of the rotational structures in Fig. 1(a) with that of Fig 1(b) is valid for the simple canonical flow, it should not be viewed as a unique relationship.

A detailed review of the dynamics of Eqns. 5 and 6 which govern these relationships is beyond the scope of this work (see for example Nomura and $\operatorname{Lin}^{16}$ and Moisy and Jiménez ${ }^{17}$ ). However, a few simple observations can be made to aid in visualization of the fields. Firstly, high-intensity (fine scale) vorticity and strain-rate structures tend to be geometrically different. While vorticity tends to concentrate into 'tube'- or 'worm'-like structures at the small scale, strain-rate structures tend to be both amorphous ('blob'-like) and 'sheet'-like. Secondly, vorticity and strain-rate structures may be spatially distinct or may overlap. Hence, the same parcel of fluid may contain different information depending on how it is viewed and isolating the important turbulent structures is necessary. Thirdly, the flame attenuates both forms of turbulent structure as they pass through. In the case of vorticity, the baroclinic torque (third term on the right of Eqn. 5) decreases the strength of the incoming vortices and may attenuate them completely. Furthermore, flamegenerated vorticity of opposite sign to the incoming vortices may be generated in the products. This process is apparent in the turbulence-flame interaction of Sec. V. The flame also acts to attenuate strain-rate structures. However, there does not appear to be any flame-generated strain rate in the products. Studies of canonical interactions between strain-rate structures and a flame front are needed to better understand the processes involved. Finally, while the flame affects the vorticity only within the gas expansion region, there is a source of fluid-dynamic strain rate associated with the flame that can manifest itself a distance from the front itself. This source appears as the pressure Hessian (fourth term on the right of Eqn. 6) and may be influential in phenomena such as the hydrodynamic instability.

\section{Diagnostics and Experiment}

In order to investigate the effects of various turbulent structures on a flame, detailed measurements of the temporally evolving turbulent flow field and flame front position were required. These measurements were obtained using the Cinema-Stereoscopic Particle Image Velocimetry (CS-PIV) ${ }^{21}$ system shown in Fig. 3. The diagnostics employ a dual forward scatter angular stereoscopic configuration. For the current experiment, the CS-PIV was operated at $1111 \mathrm{~Hz}$, producing three-component vector fields every $0.9 \mathrm{~ms}$. The field of view was $12.8 \mathrm{~mm}$ x $18.2 \mathrm{~mm}$ and the 16 pixel interrogation box used corresponded to $280 \mu \mathrm{m}$. With a $50 \%$ interrogation box overlap, this provided vectors every $140 \mu \mathrm{m}$. The flow was seeded with sub-micron $\mathrm{TiO}_{2}$ that survived passage through the flame front. Adjusting seed levels in real time allowed accurate vectors to 


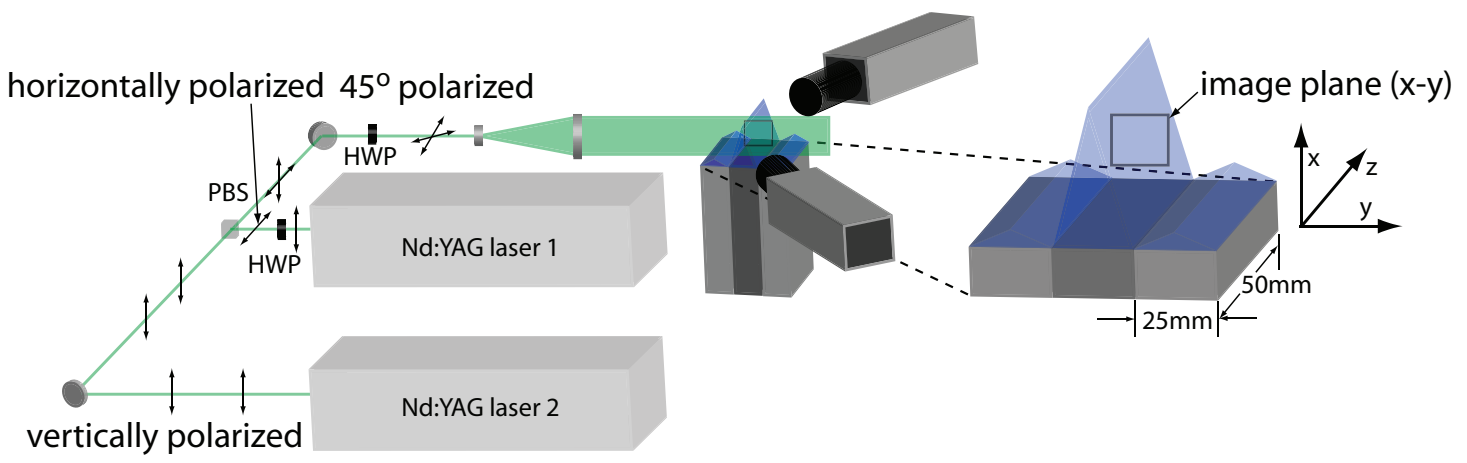

Figure 3. Experimental configuration containing CS-PIV system and burners. HWP - half wave plate, PBS polarizing beam splitter.

be computed in both the reactants and products simultaneously. The number of spurious vectors was less than $1.5 \%$ in both states.

The position of the flame front was determined by observing the dilation of the gas. This manifested itself as a significant drop in PIV particle image density. A two step predictor-corrector scheme was used to identify the contour of maximum particle density gradient. Using simultaneous CH-PLIF/Mie scattering diagnostics, this contour was shown to agree well with the true location of maximum gas density gradient. ${ }^{21}$

The flame studied was stabilized on a 2D slot Bunsen burner. In order to minimize both thermo-diffusive and preferential-diffusive effects on the flame speed, a methane-air mixture at an equivalence ratio $(\phi)$ of 0.70 was selected. The Lewis number of the deficient reactant in this mixture is approximately unity. While there is significant scatter in measured Markstein numbers, ${ }^{22}$ the $\phi=0.70$ case falls within the commonly reported range of $M a \approx 0$ for methane-air flames, indicating that preferential diffusion effects are small. Hence, the propagation speed of the flame was expected to remain relatively constant, irrespective of the local stretch rate.

The experimental geometry consisted of the three slot burners shown in Fig. 3. The center burner, which anchored the Bunsen flame of interest, had a bulk velocity of $1 \mathrm{~m} / \mathrm{s}$. The Reynolds number based on the burner width and centerline velocity was approximately 2000. The outer burners stabilized short flat flames, which provided parallel streams of hot products. Flow rates to the outer burners were adjusted to eliminate shear layers between the products of the central flame and the surrounding fluid. Turbulence was generated by passing the flow through a slot grating followed by a wire mesh. The grating generated the majority of the turbulent fluctuations with no mean velocity in the $z$-direction over the full burner length. The mesh served to break up the large, low-speed regions from the grating, preventing flashback. The turbulence intensity, defined as the ratio between the root-mean-squared velocity fluctuations in the $x$-direction at the origin and the laminar burning velocity, was 2.3. The turbulence integral scale at the origin relative to the flame diffusive thickness was 11.5. A detailed characterization of the flame is provided by Steinberg et al. ${ }^{21}$

\section{Flame Strain Rate Calculations}

Accurate computation of the strain rate on a flamelet from the experimental data required careful consideration. With the present diagnostics, measurements were only available in a single plane. Hence, only in-plane gradients of the three velocity components could be determined. The out-of-plane component of the flame normal vector, $\hat{n}$, also was unknown. Furthermore, the presence of the flame front can cause significant lag in the PIV particles due to thermophoretic and acceleration effects. This lag can introduce errors in the velocities computed within the flame front, which would translate to errors in the velocity derivatives and $a_{t}$. However, proper choice of the flame iso-surface at which the strain rate was computed, along with the use of stereo diagnostics reduced these uncertainties to acceptable levels as described below.

In order to eliminate the effects of particle lag on the measured velocities, strain rate measurements were made at the leading edge of the flame where the velocity and temperature gradients were small. Hence, strain rates were measured on a contour separated from the maximum density gradient contour by a distance of $0.65 \mathrm{~mm}$ towards the reactants in the normal direction. This corresponds to approximately $110 \%$ of the 
distance between the location of maximum density gradient and the leading edge of the flame as computed by Chemkin using the GRIMech 3.0 mechanism. Furthermore, at the computed leading edge contour the density was essentially constant, changing by approximately $1 \%$ over $0.1 \mathrm{~mm}$. This corresponded to a velocity derivative of less than $25 \mathrm{~s}^{-1}$, which is significantly less than the strain rates exerted on the flame (see Sec. $\mathrm{V})$. Hence, the flow field at the $0.65 \mathrm{~mm}$ offset contour was considered to be constant density and the divergence term in Eqn. 4 was ignored.

In order to minimize the effects of the unknown terms in the strain rate equation, the data was sampled to select isolated turbulence-flame interactions where these terms were expected to be small. Selection of the leading edge iso-contour allowed cases with minimal out-of-plane gradients of $w$ to be identified using the $\nabla \cdot \vec{u}=0$ criterion. That is, sample time periods were selected in which:

$$
\frac{\left|\frac{\partial u}{\partial x}+\frac{\partial v}{\partial y}\right|}{\left|\frac{\partial u}{\partial x}\right|+\left|\frac{\partial v}{\partial y}\right|} \leq \epsilon
$$

Hence, assuming that the out-of-plane flame normal component was approximately equal to the in-plane components, the contribution of the $n_{z}^{2} \partial w / \partial z$ term was less than $\epsilon$ of the total. A value of $\epsilon=0.1$ is used for this study. Furthermore, it is expected that this orientation component, $n_{z}$, was significantly less than $n_{x}$ or $n_{y}$ in the analyzed interactions as is explained below.

The two remaining components of $a_{t}\left(n_{1} n_{3} S_{13}\right.$ and $\left.n_{2} n_{3} S_{23}\right)$ required further consideration. These terms contain the in-plane derivatives of $w$, which can be expressly computed. However, they also contain $n_{3}$ and the out-of-plane derivatives of $u$ and $v$, which cannot. In order to minimize these terms, time intervals were selected where:

$$
\left|\frac{\partial w}{\partial x_{i}}\right|<\epsilon\left|\frac{\partial u_{j}}{\partial x_{k}}\right|
$$

where $i, j, k=1,2$, thus ensuring that the in-plane derivatives of $w$ were small. While this does not guarantee small shear strain, assuming that out-of-plane derivatives of $u$ and $v$ are distributed around zero, their net effect should statistically be small if a large number of events are analyzed.

An additional condition for the time intervals investigated was set by the magnitude of $w$, which was available from the stereoscopic measurements. For any interaction event to be studied, it was necessary that there was little out-of-plane convection. Such convection would appear as erroneous evolution of both the turbulent structure and flame shape. Hence, time periods were selected during which $|w|<\epsilon|\vec{u}|$.

The effects of the unknown fluid strain rates $\left(S_{i 3}\right)$ are further minimized if the out-of-plane orientation of the flame surface is small. While this orientation cannot be explicitly measured, it is expected to be small relative to the in-plane orientations during interactions adhering to the above restrictions. In the laminar case, the flame studied would have $n_{z}=0$; the slot burner would produce a 2D flame invariant in the $z$ direction. Non-zero values in the turbulent flame are associated with regions out-of-plane curvature. Such regions induce both significant values of $w$ and $\partial w / \partial z$ due to the hydrodynamic instability. ${ }^{23}$ Hence, by adhering to the above criteria for the velocity field, turbulence-flame interactions are implicitly selected in which the flame wrinkling is largely in the measurement plane. This results in low values of $n_{z}$ and further reduces the effects of the out-of-plane strain rates. However, fully three-dimensional measurements are required to assess these assumptions (e.g. Steinberg et al. ${ }^{24}$ ).

The presence of the flame front also required careful design of the numerical method used to compute $a_{t}$. Velocity data were measured on a regular square grid while the flame was defined freely in space. Hence, velocity data had to be interpolated to the flame surface. At the flame density iso-surface selected, neither gas acceleration due to expansion or thermophoretic particle lag effected the measured velocities. However, significant error to the numerical derivatives could be induced due to the proximity of this expansion related high acceleration region. It was therefore necessary to both interpolate velocities and construct derivatives using selected velocity nodes that often formed irregular patterns. Observation showed that the leading edge of the gas acceleration through the flame was always greater than $100 \mu \mathrm{m}$ downstream of the selected flame contour. Hence, usable nodes on the downstream side of the flame contour were restricted to those within $100 \mu \mathrm{m}$. All points on the upstream side were usable. This irregular node pattern and freely defined flame required a triangular mesh on which to compute the velocity derivatives. These derivatives were computed at the triangle node corresponding to the flame using a second order accurate inverse distance weighted method based on the gradients over the faces of the adjacent triangles. ${ }^{25}$ 


\section{Results and Discussion}

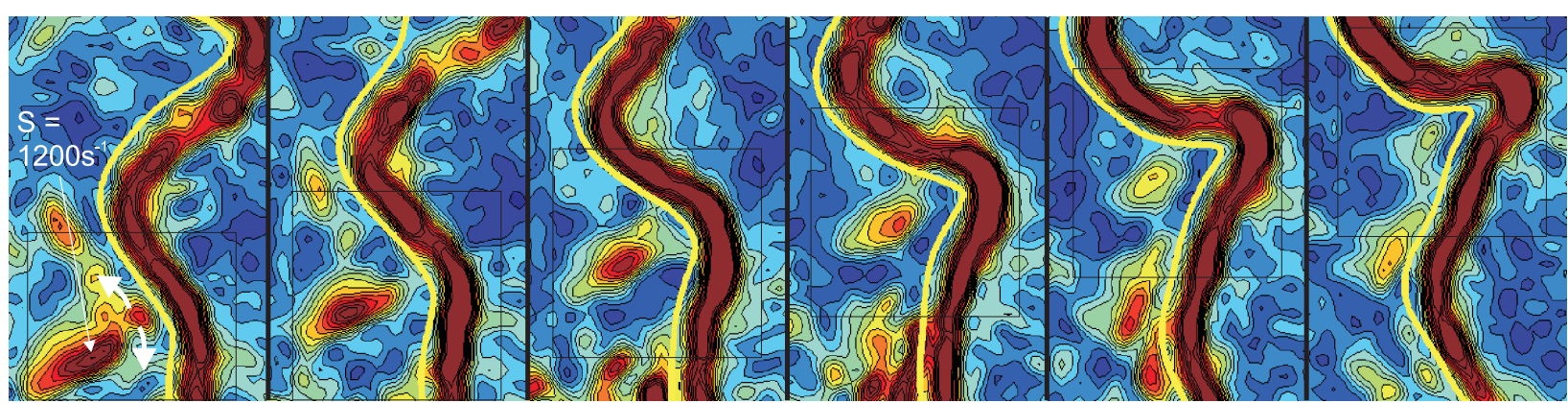

(a) Contours of strain rate $(S)$ between $0 s^{-1}$ and $1200 s^{-1}$. Flame contour in yellow.

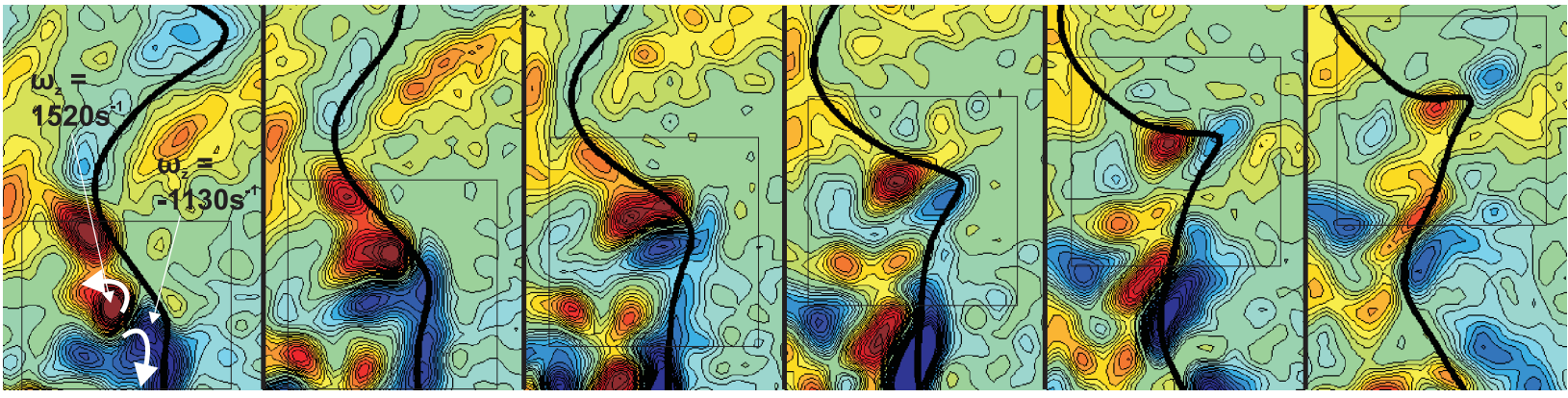

(b) Contours of vorticity $\left(\omega_{z}\right)$ between $-1200 s^{-1}$ (blue) and $1200 s^{-1}$ (red). Flame contour in black.

Figure 4. Interaction 1 - A strong strain-rate structure and strong vortical structures interact with the flame. Considerable flame surface area is generated and a large wrinkle forms. Field of view is $6 \mathrm{~mm} x 9 \mathrm{~mm}$, time between frames is $0.9 \mathrm{~ms}$. Reactants on left. Bulk flow is upward.

The analysis of Sec. II indicates that the turbulent structures responsible for the generation of flame surface area are those associated with concentrated fluid dynamic strain rate, not vorticity. The interaction between fluid with high vorticity and negligible strain rate should have no significant effect on the flame surface area. To demonstrate this, Figs. 4-6 (Interactions 1-3) present the interaction between various turbulent structures and the flame front. In all cases, strain-rate structures $(S=$ resolved component of $\left(S_{i j} S_{i j}\right)^{1 / 2}$, subfigure $\left.a\right)$ and vortical structures $\left(\omega_{z}\right.$, subfigure $\left.b\right)$ are shown separately. Additionally, Fig. 7 (a) shows the temporal evolution of the maximum $a_{t}$ for each interaction, while Fig. 7(b) shows $a_{t}$ profiles along the flame surface at selected times. In both cases only the flame segments in the boxed regions of Figs. 4-6 were considered. It should be noted that the flame front manifests itself in the $S$ fields as a region of high strain due to the acceleration of the gas through the flame (i.e. $\nabla \cdot \vec{u}>0$ ). However, this strain rate is not a result of the turbulence and hence not the focus of this work. Nevertheless, the high acceleration region is an indicator of the flame thickness and the accuracy of the leading edge isocontour.

During Interaction 1, a strong strain-rate structure (Fig. 4(a)) and an associated pair of vortices (Fig. 4(b)) interacted with the flame. The maximum value of $S$ was $1200 \mathrm{~s}^{-1}$, while the vorticity peaks were $1520 s^{-1}$ and $-1130 s^{-1}$. From Fig. $7($ a) it is apparent that this interaction exerted a high magnitude strain rate on the flame for an extended period. This produced considerable flame surface area, which was wrapped around the pair of vortices forming a large wrinkle. The long duration of the strain rate on the flame corresponds to the long residence time of the strain-rate structure. In contrast, Interaction 2 (Fig. 5) involved vortical structures of greater strength $\left(1730 s^{-1}\right.$ and $-1810 s^{-1}$ peak vorticity) and a similar strainrate structure $\left(1220 \mathrm{~s}^{-1}\right.$ peak $\left.S\right)$. However, the strain-rate structure was attenuated much more quickly than in Interaction 1. As a consequence Fig. 7(a) shows that $a_{t}$ for this interaction quickly decreased. Hence, this interaction produced less flame surface area than Interaction 1 and a smaller wrinkle was formed. Finally, Interaction 3 (Fig. 6) contained similar vortical structures to the previous two. However, the configuration was such that the strain-rate structure was not adjacent to the flame when the vortices begin to interact. By the time the strain-rate structure reached the flame, it was nearly attenuated. The resultant $a_{t}$ in Fig. 7(a) was low and nearly no flame surface area was created. Furthermore, the geometry of the flame and vortices 


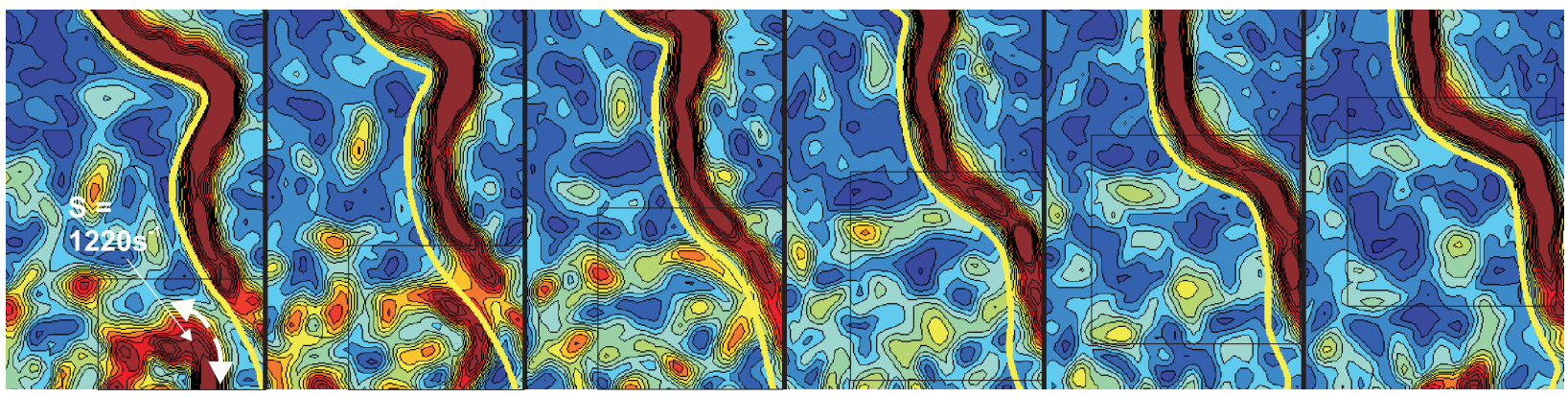

(a) Contours of strain rate $(S)$ between $0 s^{-1}$ and $1200 s^{-1}$. Flame contour in yellow.
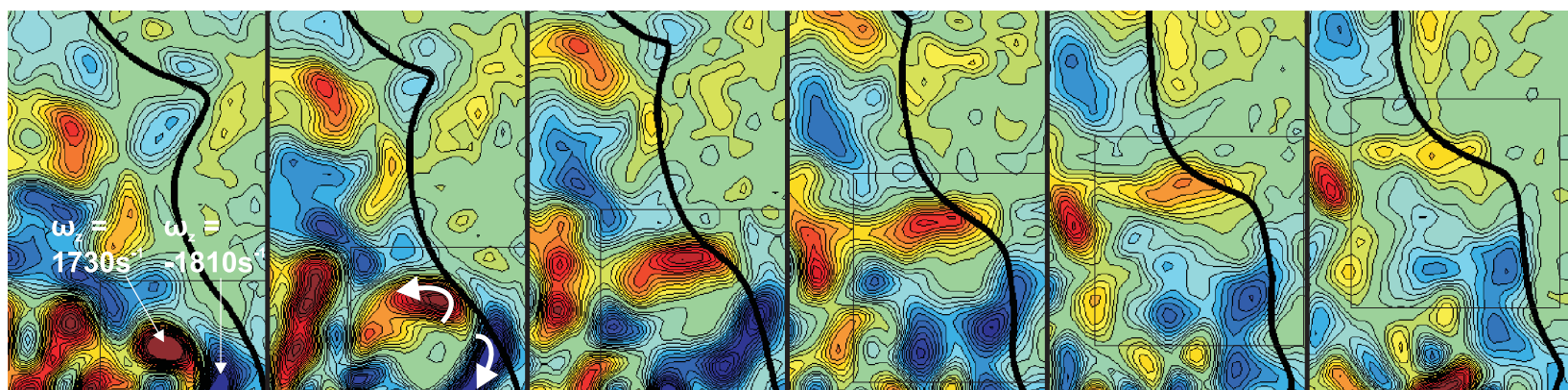

(b) Contours of vorticity $\left(\omega_{z}\right)$ between $-1200 s^{-1}$ (blue) and $1200 s^{-1}$ (red). Flame contour in black.

Figure 5. Interaction 2 - A strong strain-rate structure and strong vortical structures interact with the flame. The strain-rate structure is quickly attenuated. A moderate amount of flame surface is generated and a medium sized wrinkle forms. Field of view is $6 \mathrm{~mm} \times 9 \mathrm{~mm}$, time between frames is $0.9 \mathrm{~ms}$. Reactants on left.

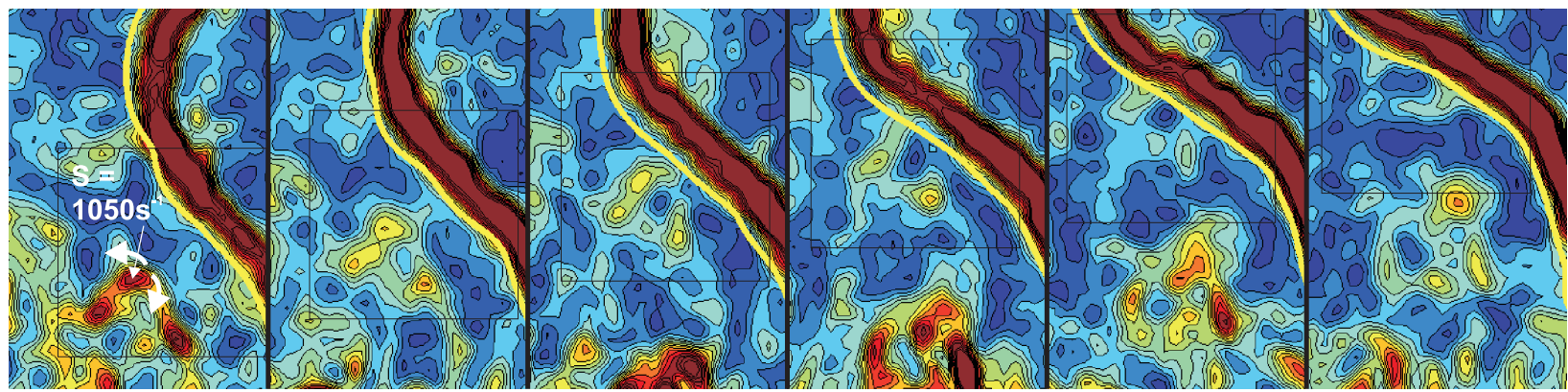

(a) Contours of strain rate $(S)$ between $0 \mathrm{~s}^{-1}$ and $1200 \mathrm{~s}^{-1}$. Flame contour in yellow.

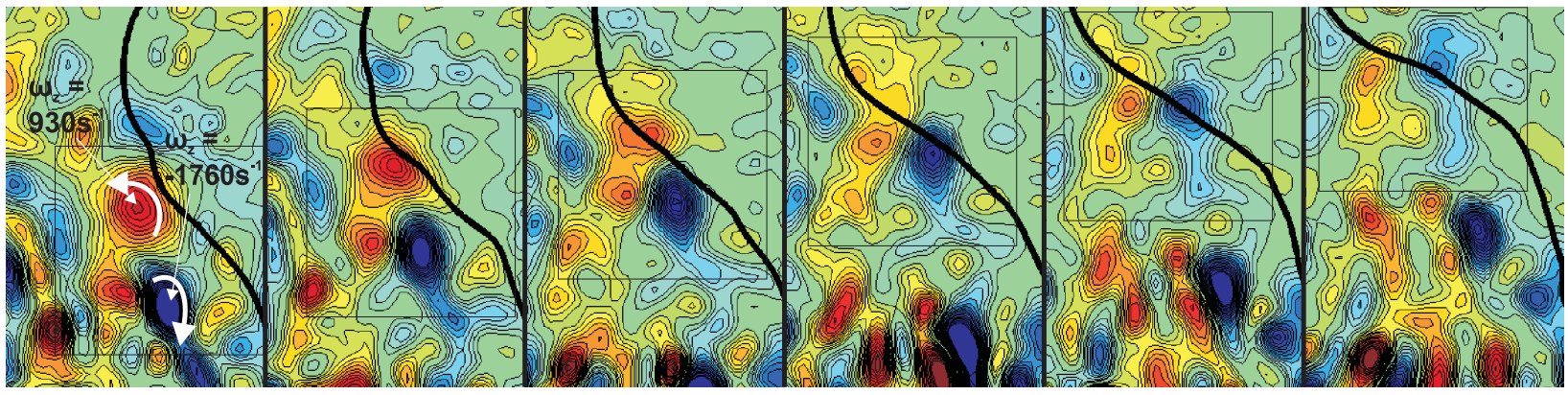

(b) Contours of vorticity $\left(\omega_{z}\right)$ between $-1200 s^{-1}$ (blue) and $1200 s^{-1}$ (red). Flame contour in black.

Figure 6. Interaction 3 - Strong vortical structures interact with the flame. The strain-rate structure is attenuated before interacting. No flame surface is generated and no wrinkle is formed. Field of view is $6 \mathrm{~mm}$ x $9 \mathrm{~mm}$, time between frames is $0.9 \mathrm{~ms}$. Reactants on left. 
was such that the flame surface was not wrinkled; the flame was already oriented around the vortices and the interaction had little effect. Hence, it is apparent that the interaction between turbulence and a flame front is a simultaneous process of strain-rate structures generating flame surface area and vortical structures redistributing it into wrinkles.

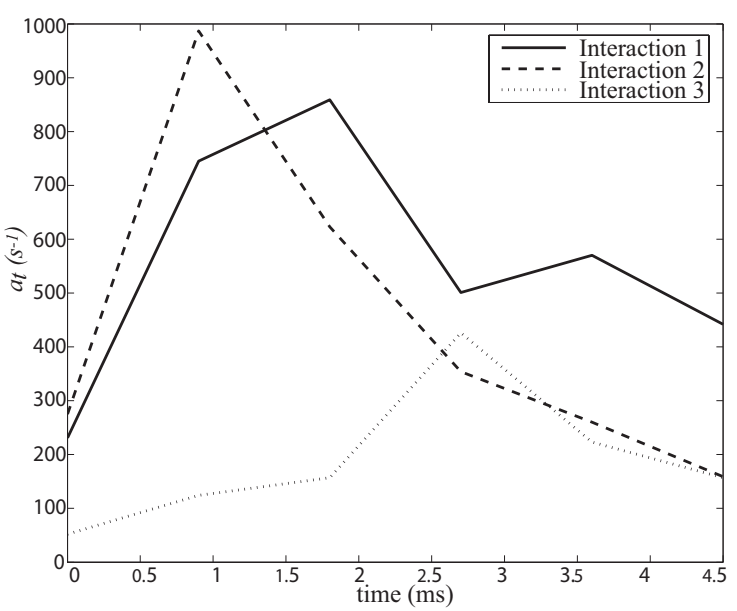

(a) Temporal evolution of the strain rate on the flame surface $\left(a_{t}\right)$.

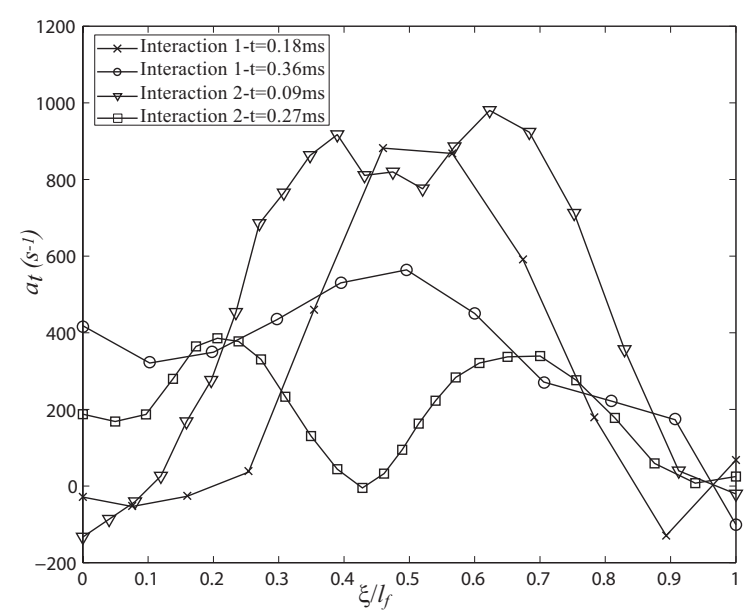

(b) Flame strain rate $\left(a_{t}\right)$ profiles measured along the flame surface at various times.

Figure 7.

Figure $7(\mathrm{~b})$ shows the distribution of strain rate on the flame surface at select times. At each time, only the flame surface in the boxed region is considered. The horizontal coordinate is the distance along the flame surface $(\xi)$ normalized by the length of the flame segment $\left(l_{f}\right)$ in the box. As can be seen, the location of the flame strain rate coincides with the location of the strain-rate structures. For example, Interaction 1 contains a single peak of strain rate throughout, while Interaction 2 develops two peaks coinciding with the locations of the split strain-rate structures at $t=0.27 \mathrm{~ms}$ (fourth frame of Fig. 5). Hence, the straining of the flame surface is well characterized by the strength, location, and residence time of the strain-rate structures.

This concept of simultaneous straining and wrinkling of flame surface by different structures allows the complex turbulence-flame interactions encountered in practical flows to be analyzed; the assumption that the turbulence exists as isolated counter-rotating vortex pairs is unnecessary. For example, consider the interaction shown in Fig. 8. In this case, there are several vortical structures interacting with the flame simultaneously. This geometry is not considered in the canonical configuration. However, when viewed in terms of strain rate, there are several distinct strain-rate structures, each of which interacts with the flame in isolation. Furthermore, significant strain on the flame is exerted by only two structures and flame surface area is generated at these locations. The resultant wrinkle forms around the group of vortices associated with the two significant strain-rate structures.

While the flame straining and wrinkling processes are continuously and simultaneously occurring, it is possible to largely isolate each mechanism to observe their distinct effects. Interactions demonstrating these mechanisms are shown in Figs. 9 and 10. The resulting maximum $a_{t}$ and flame curvature $(C)$ are shown in Fig. 11. Figure 9 (Interaction 5) shows the interaction between two strain-rate structures and the flame front. The vortical structures in this interaction were configured such that no flame wrinkle was produced; the flame surface was already oriented around the vortices. However, the extensively straining structures still exerted strain on the flame surface, generating surface area and resulting in a longer flat flame contour. This is confirmed by Fig. 11. In Fig. 11(a), two pulses of $a_{t}$ are exerted on the flame corresponding to the two strain-rate structures. However, the turbulence does not generate a wrinkle. Figure 11(b) shows that the initial slightly positive curvature is stretched out, resulting in a flatter flame front. This process is analogous to the counterflow geometry of Fig. 2(b).

Conversely, Fig. 10 (Interaction 6) shows a vortex pair interacting with the flame. The structure with positive vorticity is considerably stronger than its counterpart. The strain rate structure is also weak. As the positive vortex encounters the flame, existing flame surface is wrapped around it The weaker, negative 


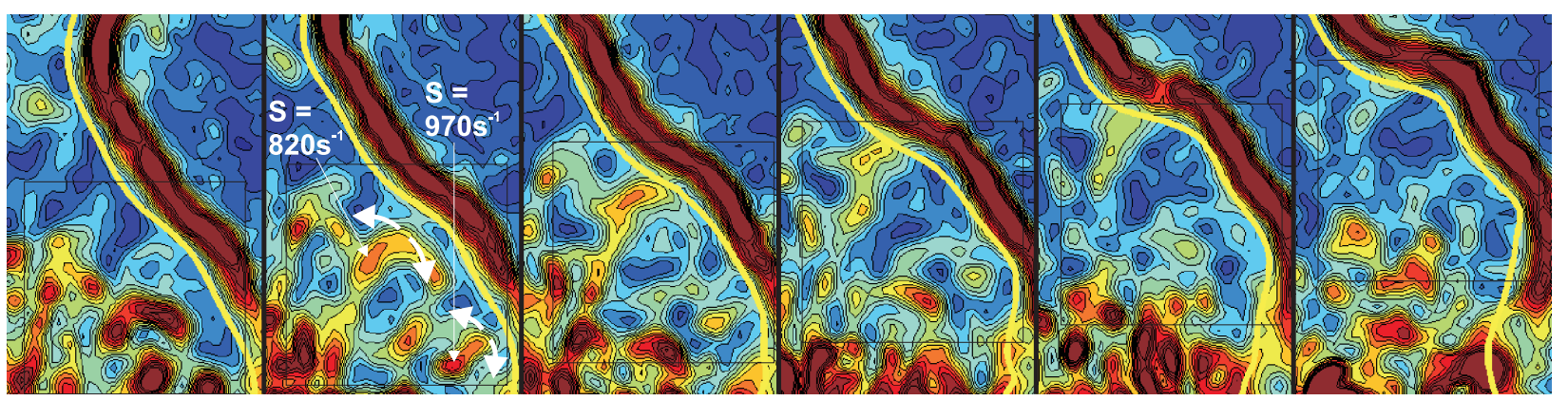

(a) Contours of strain rate $(S)$ between $0 s^{-1}$ and $1200 s^{-1}$. Flame contour in yellow.

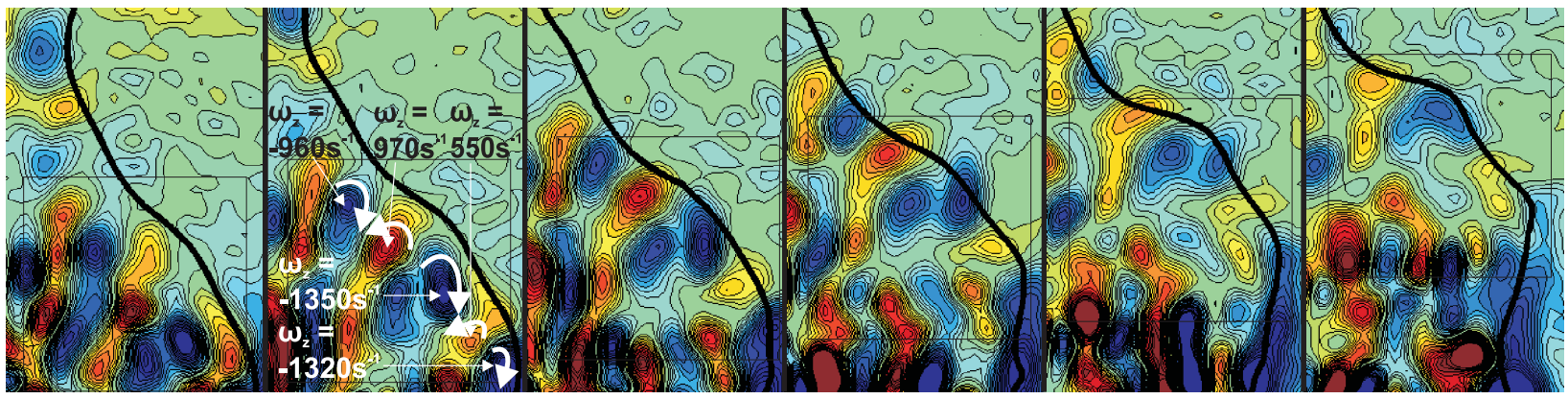

(b) Contours of vorticity $\left(\omega_{z}\right)$ between $-1200 s^{-1}$ (blue) and $1200 s^{-1}$ (red). Flame contour in black.

Figure 8. Interaction 4 - A complicated vorticity field interacts with the flame. However, the generation of flame surface area is easily understood in terms of the strain-rate structures. Field of view is $6 \mathrm{~mm} \times 9 \mathrm{~mm}$, time between frames is $0.9 \mathrm{~ms}$. Reactants on left.

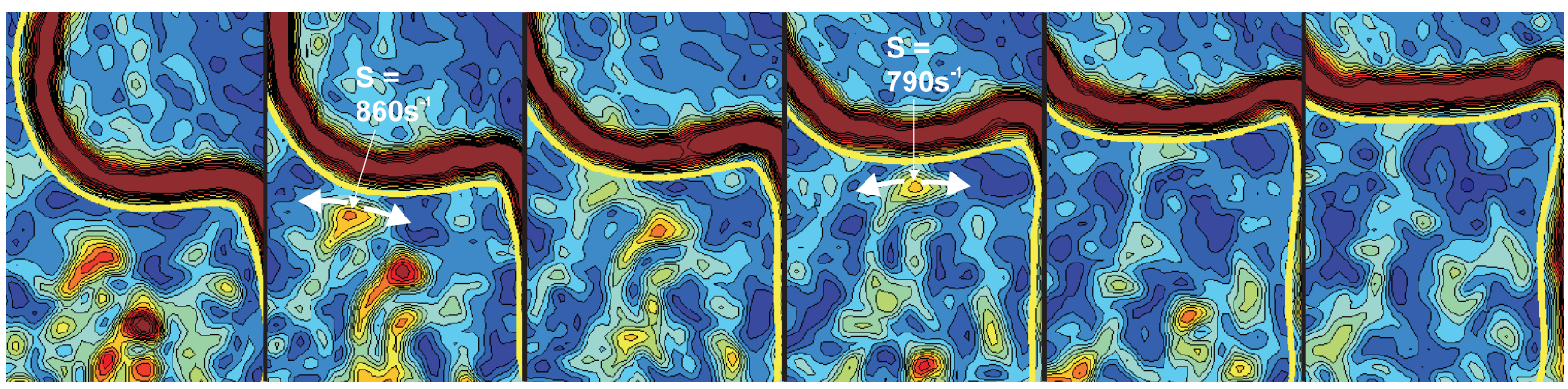

(a) Contours of strain rate $(S)$ between $0 s^{-1}$ and $1200 s^{-1}$. Flame contour in yellow.

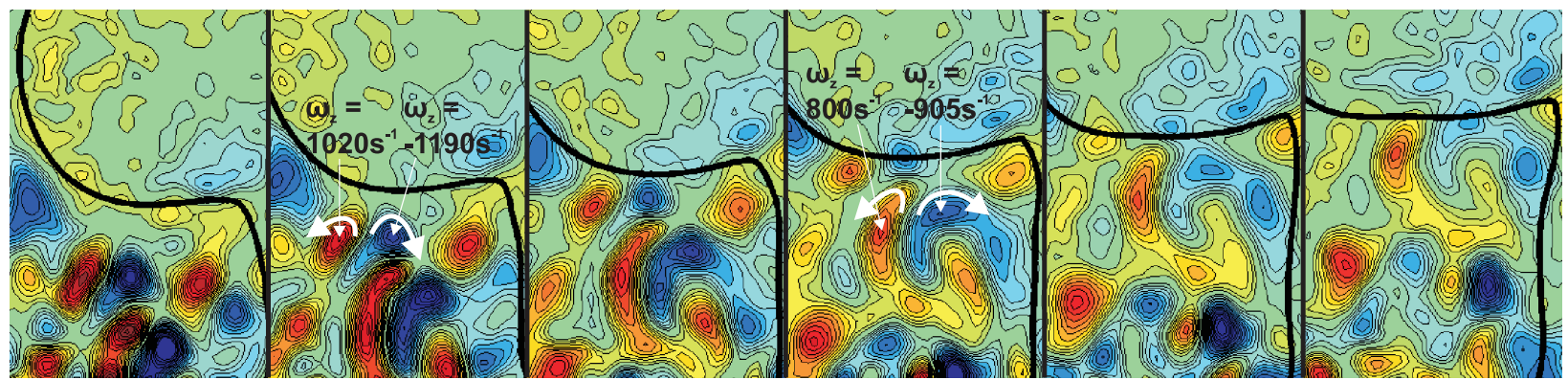

(b) Contours of vorticity $\left(\omega_{z}\right)$ between $-1200 s^{-1}$ (blue) and $1200 s^{-1}$ (red). Flame contour in black.

Figure 9. Interaction 5 - Strain-rate structures elongating a flat flame segment. Flame surface area is generated without a wrinkle begin formed. Field of view is $6 \mathrm{~mm} \times 9 \mathrm{~mm}$, time between frames is $0.9 \mathrm{~ms}$. Reactants on left. 


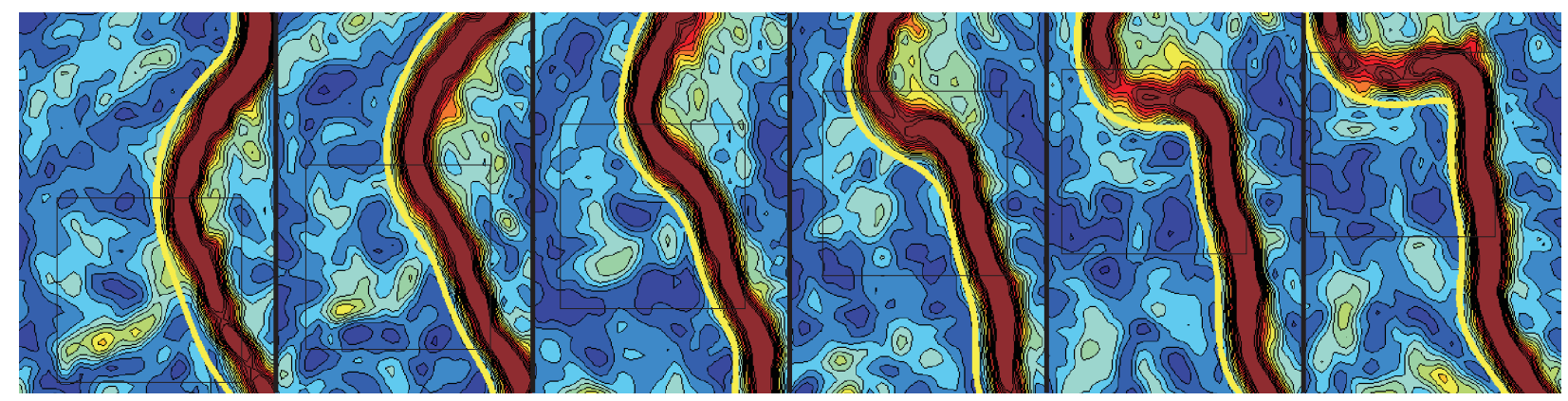

(a) Contours of strain rate $(S)$ between $0 s^{-1}$ and $1200 s^{-1}$. Flame contour in yellow.

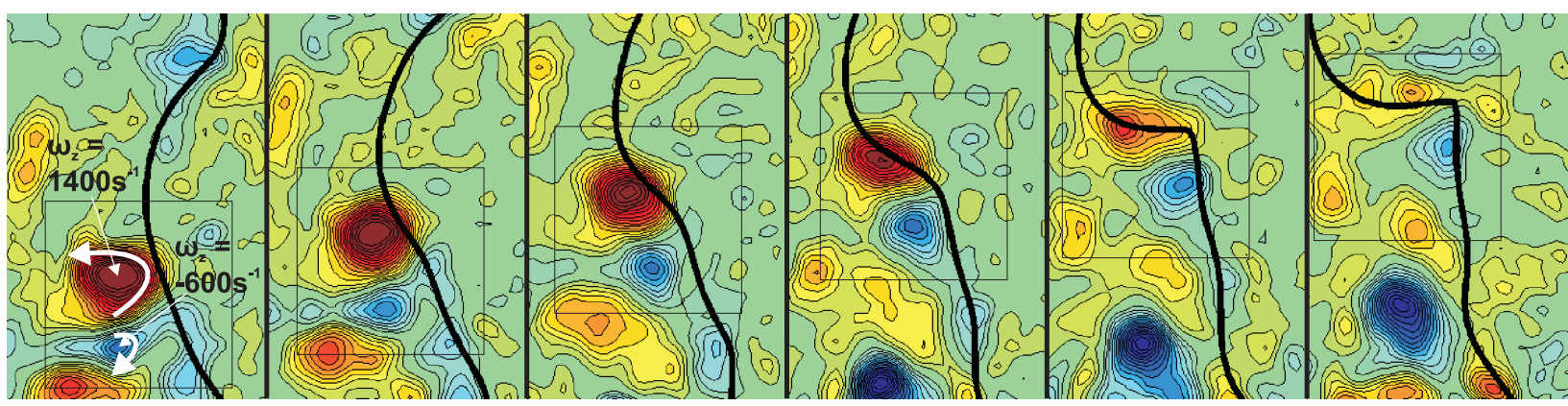

(b) Contours of vorticity $\left(\omega_{z}\right)$ between $-1200 \mathrm{~s}^{-1}$ (blue) and $1200 \mathrm{~s}^{-1}$ (red). Flame contour in black.

Figure 10. Interaction 6 - A strong vortical structure wraps existing flame surface around it. Little flame surface area is generated. Field of view is $6 \mathrm{~mm} \times 9 \mathrm{~mm}$, time between frames is $0.9 \mathrm{~ms}$. Reactants on left.

vortex similarly influences the flame, but to a much lesser extent and a non-symmetric wrinkle is formed. However, due to the weak strain rate structure, the $a_{t}$ exerted on the flame is relatively low throughout the interaction as shown in Fig. 11(a). The flame surface is wrapped up by the vortices resulting in a negative curvature wrinkle (Fig. 11(b)). Hence, this wrinkle is formed without significant straining of the flame surface.

\section{Conclusions}

The interaction between a turbulent flow and a premixed flame has been studied experimentally using Cinema-Stereoscopic PIV (CS-PIV). This diagnostic captures the evolution of the flow field and flame front position with high spatial and temporal resolution. A methane-air slot Bunsen flame with coflowing flat pilot flames was studied. The equivalence ratio was 0.7 and the bulk velocity was $1 \mathrm{~m} / \mathrm{s}$.

Analysis of the strain rate term of the flame surface density equation indicated that the turbulent structures responsible for the generation of flame surface area are those of concentrated fluid dynamic strain rate, not vorticity. This is in contrast to the canonical idea of flame wrinkling by vortical structures. A new interpretation of the wrinkling process involving the simultaneous stretching of the flame by turbulent strain-rate structures and redistribution of the flame surface into wrinkles by vortical structures was presented.

This interpretation was confirmed using the CS-PIV measurements. It was shown that the generation of large strain rates on the flame corresponded to interactions involving strong strain-rate structures interacting with the flame for extensive time periods. As the strength or interaction time of the strain-rate structures decreased, the amount of flame surface generated and the size of the flame wrinkles also decreased. Hence, the turbulent strain-rate structures provide a good characterization for the strength, duration, and location of the flame surface generation. Furthermore, it was shown that the new interpretation of the straining and wrinkling process is more versatile than the canonical interpretation. A complex flow involving many closely spaced vortical structures was observed. This geometry is not considered by the canonical configuration. However, when viewed in terms of strain-rate structures, the complex geometry devolves into simple interactions between isolated structures and the flame. The effects of strain-rate structures and vortical structures in isolation also were observed. The strain-rate structures interacted with the flame in a manner similar to 


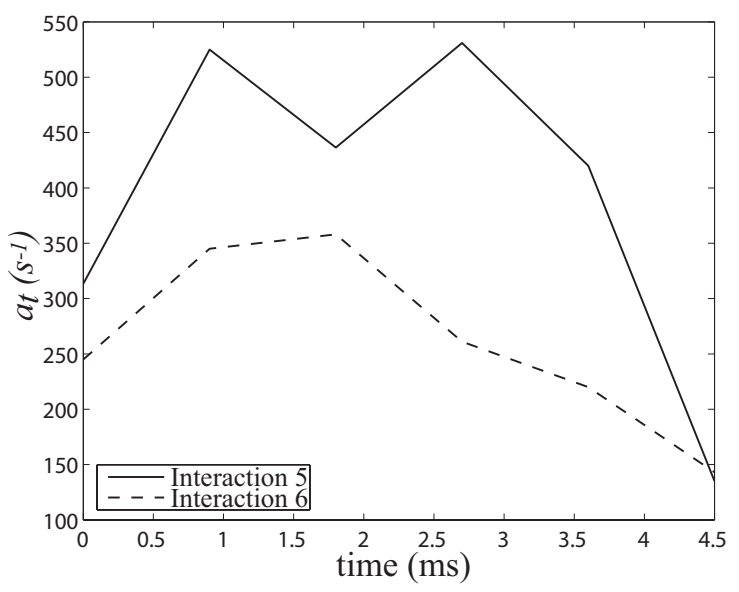

(a) Strain rate.

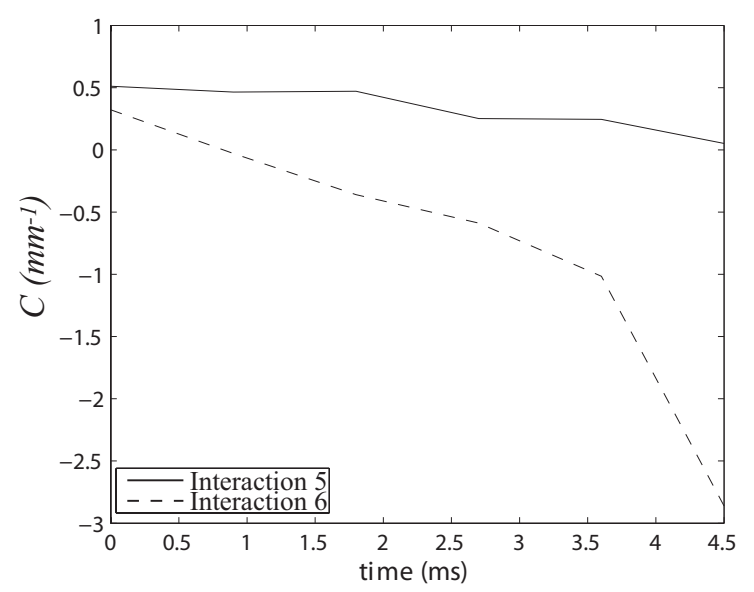

(b) Flame curvature.

Figure 11. Temporal evolution of the maximum strain rate and flame curvature for Interactions 5 and 6.

that of a counterflow flame. Flame surface area appeared to be generated without a wrinkle being formed; the planar flame segment was stretched and elongated. The vortical structures wrapped the flame around themselves, creating a wrinkle without appearing to generate flame surface area.

\section{Appendix}

A general transport equation for the strain rate components in a reacting flow can be derived by taking the spatial derivative the equations for conservation of momentum:

$$
\frac{\partial}{\partial x_{j}} \frac{\partial u_{i}}{\partial t}+\frac{\partial}{\partial x_{j}}\left(u_{k} \frac{\partial u_{i}}{\partial x_{k}}\right)=\frac{\partial g}{\partial x_{j}}-\frac{\partial}{\partial x_{j}}\left(\frac{1}{\rho} \frac{\partial p}{\partial x_{i}}\right)+\frac{\partial}{\partial x_{j}}\left(\nu \frac{\partial^{2} u_{i}}{\partial x_{k} \partial x_{k}}\right)
$$

Setting $A_{i j}=\partial u_{i} / \partial x_{j}$, this can be expanded as:

$$
\frac{\partial A_{i j}}{\partial t}+u_{k} \frac{\partial A_{i j}}{\partial x_{k}}=-A_{i k} A_{k j}-\frac{1}{\rho} \frac{\partial^{2} p}{\partial x_{i} \partial x_{j}}+\frac{1}{\rho^{2}} \frac{\partial p}{\partial x_{i}} \frac{\partial \rho}{\partial x_{j}}+\nu \frac{\partial^{2} A_{i j}}{\partial x_{k} \partial x_{k}}+\frac{\partial \nu}{\partial x_{j}} \frac{\partial^{2} u_{i}}{\partial x_{k} \partial x_{k}}
$$

Recognizing that $S_{i j}=1 / 2\left(A_{i j}+A_{j i}\right)$, the appropriate forms of Eqn. 10 can be summed to yield:

$$
\begin{aligned}
\frac{\partial S_{i j}}{\partial t}+u_{k} \frac{\partial S_{i j}}{\partial x_{k}} & =-\left(\frac{A_{i k} A_{k j}+A_{j k} A_{k i}}{2}\right)-\frac{1}{\rho} \frac{\partial^{2} p}{\partial x_{i} \partial x_{j}}+\nu \frac{\partial^{2} S_{i j}}{\partial x_{k} \partial x_{k}} \\
& +\frac{1}{2 \rho^{2}}\left(\frac{\partial p}{\partial x_{i}} \frac{\partial \rho}{\partial x_{j}}+\frac{\partial p}{\partial x_{j}} \frac{\partial \rho}{\partial x_{i}}\right)+\frac{1}{2}\left(\frac{\partial \nu}{\partial x_{j}} \frac{\partial^{2} u_{i}}{\partial x_{k} \partial x_{k}}+\frac{\partial \nu}{\partial x_{i}} \frac{\partial^{2} u_{j}}{\partial x_{k} \partial x_{k}}\right)
\end{aligned}
$$

The first term on the right can be written in terms of $\underline{S}$ and $\vec{\omega}$ by recognizing that $A_{i j}=S_{i j}-\Omega_{i j}$ and equating the terms of $\underline{\Omega}$ with the various vorticity components. This yields the final transport equation:

$$
\begin{aligned}
\frac{\partial S_{i j}}{\partial t}+u_{k} \frac{\partial S_{i j}}{\partial x_{k}} & =-S_{i k} S_{k j}-\frac{1}{4}\left(\omega_{i} \omega_{j}-\delta_{i j} \omega_{k} \omega_{k}\right)-\frac{1}{\rho} \frac{\partial^{2} p}{\partial x_{i} \partial x_{j}}+\nu \frac{\partial^{2} S_{i j}}{\partial x_{k} \partial x_{k}} \\
& +\frac{1}{2 \rho^{2}}\left(\frac{\partial p}{\partial x_{i}} \frac{\partial \rho}{\partial x_{j}}+\frac{\partial p}{\partial x_{j}} \frac{\partial \rho}{\partial x_{i}}\right)+\frac{1}{2}\left(\frac{\partial \nu}{\partial x_{j}} \frac{\partial^{2} u_{i}}{\partial x_{k} \partial x_{k}}+\frac{\partial \nu}{\partial x_{i}} \frac{\partial^{2} u_{j}}{\partial x_{k} \partial x_{k}}\right)
\end{aligned}
$$

\section{References}

${ }^{1}$ Marble, F. E. and Broadwell, J. E., "The coherent flame model for turbulent chemical reactions," Tech. Rep. TRW-9-PU, Project Squid, 1977.

${ }^{2}$ Choi, C. R. and Huh, K. Y., "Development of a coherent flamelet model for a spark ignited turbulent premixed flame in a closed vessel," Combust. Flame, Vol. 114, No. 3, 1998, pp. 336-348. 
${ }^{3}$ Hawkes, E. R. and Cant, R. S., "A flame surface density approach to Large Eddy Simulation of premixed turbulent combustion," Proc. Combust. Inst., Vol. 28, 2000, pp. 51-58.

${ }^{4}$ Charlette, F., Meneveau, C., and Veynante, D., "A power-law flame wrinkling model for LES of premixed turbulent combustion. Part 1: non-dynamic formulation and initial tests," Combust. Flame, Vol. 131, No. 2, 2002, pp. 159-180.

${ }^{5}$ Fureby, C., "A fractal flame-wrinkling large eddy simulation model for premixed turbulent combustion," Proc. Combust. Inst., Vol. 30, 2005, pp. 593-601.

${ }^{6}$ Poinsot, T., Veynante, D., and Candel, S., "Quenching processes and premixed turbulent combustion diagrams," J. Fluid Mech., Vol. 228, 1991, pp. 561-606.

${ }^{7}$ Meneveau, C. and Poinsot, T., "Stretching and quenching of flamelets in premixed turbulent combustion," Combust. Flame, Vol. 86, No. 4, 1991, pp. 311-332.

${ }^{8}$ Colin, O., Ducros, F., Veynante, D., and Poinsot, T., "A thickened flame model for large eddy simulations of turbulent premixed combustion," Phys. Fluids, Vol. 12, No. 7, 2000, pp. 1843-1863.

${ }^{9}$ Mueller, C. J., Driscoll, J. F., Reuss, D. L., Drake, M. C., and Rosalik, M. E., "Vorticity generation and attenuation as vorticies convect through a flame," Combust. Flame, Vol. 112, No. 3, 1998, pp. 342-358.

${ }^{10}$ Sinibaldi, J. O., Mueller, C. J., Tulkki, A. E., and Driscoll, J. F., "Suppression of flame wrinkling by buoyancy: the baroclinic stabilization mechanism," AIAA J., Vol. 36, No. 8, 1998, pp. 1432-1438.

${ }^{11}$ Renard, P.-H., Thevenin, D., Rolon, J., and Candel, S., "Dynamics of flame/vortex interactions," Prog. Energy Combust. Sci., Vol. 26, 2000, pp. 225-282.

${ }^{12}$ Kadowaki, S. and Hasegawa, T., "Numerical simulation of dynamics of premxied flames: flame instability and vortexflame interaction," Prog. Energy Comust. Sci., Vol. 31, 2005, pp. 193-241.

${ }^{13}$ Steinberg, A. M., Driscoll, J. F., and Ceccio, S. L., "Temporal evolution of flame stretch due to turbulence and the hydrodynamic instability," To appear: Proc. Combust. Inst., Vol. 32, 2008.

${ }^{14}$ Kolmogorov, A. N., "A refinement of previous hypotheses concerning the local structure of turbulence in a viscous incompressible fluid at high Reynolds number," J. Fluid Mech., Vol. 13, 1632, pp. 82.

${ }^{15}$ Boratav, O. N. and Pelz, R. B., "Structures and structure functions in the inertial range of turbulence," Phys. Fluids, Vol. 9, 1996, pp. 1400-1415.

${ }^{16}$ Nomura, K. K. and Diamessis, P. J., "The interaction of vorticity and rate-of-strain in homogeneous sheared turbulence," Phys. Fluids, Vol. 12, 2000, pp. 846.

${ }^{17}$ Moisy, F. and Jiménez, J., "Geometry and clustering of intense structures in isotropic turbulence," J. Fluid Mech., Vol. 513, 2004, pp. 111-133.

${ }^{18}$ Swaminathan, N. and W., G. R., "Interaction of turbulence and scalar fields in premixed flames," Phys. Fluids, Vol. 18, 2006, pp. 045102.

${ }^{19}$ Chakraborty, N. and Swaminathan, N., "Influence of the Damkoler number on turbulence-scalar interaction in premixed flames. I. Physical insight," Phys. Fluids, Vol. 19, 2007, pp. 0451003.

${ }^{20}$ Dahm, W., Scheil, C. M., and Tryggvason, G., "Dynamics of vortex interaction with a density interface," J. Fluid Mech., Vol. 205, 1989, pp. 1-43.

${ }^{21}$ Steinberg, A. M., Driscoll, J. F., and Ceccio, S. L., "Measurements of turbulent premixed flame dynamics using cinemastereoscopic PIV," Exp. Fluids, Vol. 44, 2008, pp. 985-999.

${ }^{22}$ Müller, U. C., Bollig, M., and Peters, N., "Approximations for burning velocities and Markstein numbers for lean hydrocarbon and methanol flames," Combust. Flame, Vol. 108, No. 3, 1997, pp. 349-356.

${ }^{23}$ Williams, F., Combustion Theory, 2nd ed., Addison-Wesley, Reading, USA, 1985.

${ }^{24}$ Steinberg, A. M., Driscoll, J. F., and Ceccio, S. L., "Temporally resolved pseudo 3D measurements of flame strain using orthogonal-plane cinema-stereoscopic PIV," 23rd International Congress on Theoretical and Applied Mechanics, Adelaide, Australia, 2008

${ }^{25}$ Amidror, I., "Scattered data interpolation methods for electronic imaging systems: a survey," J. Electron. Imaging, Vol. 11, 2002, pp. 157-176. 